\title{
On Multiple Users Scheduling Using Superposition Coding Over Rayleigh Fading Channels
}

\author{
Ammar Zafar, Student Member, IEEE, Mohammad Shaqfeh, Member, IEEE, Mohamed-Slim Alouini, Fellow, \\ IEEE, and Hussein Alnuweiri, Member, IEEE
}

\begin{abstract}
In this letter, numerical results are provided to analyze the gains of multiple users scheduling via superposition coding with successive interference cancellation in comparison with the conventional single user scheduling in Rayleigh blockfading broadcast channels. The information-theoretic optimal power, rate and decoding order allocation for the superposition coding scheme are considered and the corresponding histogram for the optimal number of scheduled users is evaluated. Results show that at optimality there is a high probability that only two or three users are scheduled per channel transmission block. Numerical results for the gains of multiple users scheduling in terms of the long term throughput under hard and proportional fairness as well as for fixed merit weights for the users are also provided. These results show that the performance gain of multiple users scheduling over single user scheduling increases when the total number of users in the network increases, and it can exceed $10 \%$ for high number of users.
\end{abstract}

Index Terms-Rayleigh block-fading; broadcast channels; multiple users scheduling; successive interference cancellation.

\section{INTRODUCTION}

Channel-aware opportunistic scheduling is commonly applied in current wireless communication systems in order to exploit the fading channels of the users when they are at their peak conditions. This is often referred to as multiuser diversity (MUD) gain [1]. The common scheduling scheme is to select only a single user per channel transmission block. Single user scheduling, in its generic form, is based on selecting the user with the best weighted instantaneous channel capacity, where the users' weights control the trade-off between total throughput and fairness among the network's users [2].

From information-theoretic perspective, single user (SU) selection is optimal in the special case of maximizing the sum (of all users) throughput of the system, where the user with the best channel quality gets selected [3]. However, for all other operating points of the system in which the objective is to maximize a weighted sum of the users' rates, SU selection is suboptimal. The optimal scheme in this case is superposition coding (SC) with successive interference cancellation (SIC) at the receivers. This is the capacity-achieving scheme for the degraded broadcast channel [4]. With SC, multiple users

A. Zafar and M. -S. Alouini are with the Electrical Engineering Program, King Abdullah University of Science and Technology (KAUST), Thuwal, Makkah Province, Saudi Arabia. (E-mail: \{ammar.zafar,slim.alouini\}@ kaust.edu.sa).

M. Shaqfeh and H. Alnuweiri are with the Department of Electrical and Computer Engineering, Texas A\&M University at Qatar, C/o Qatar Foundation, PO Box 23874, Doha, Qatar. (E-mail: \{Mohammad.Shaqfeh, Hussein.Alnuweiri\}@qatar.tamu.edu).

This paper was made possible by YSREP grant \# 2-011-2-002 from the Qatar National Research Fund (a member of Qatar Foundation). Furthermore, KAUST funded the efforts of A. Zafar partially and M.-S. Alouini fully. The statements made herein are solely the responsibility of the authors. are scheduled simultaneously over the same channel block, and hence the codewords of the different users interfere with each other. So, this is a non-orthogonal transmission scheme. Therefore, the rates of the individual users become less than when they are scheduled alone due to the interfering codewords of the other users. However, each user gets scheduled more frequently and hence on the long term the individual and the aggregate (i.e. sum) rates get enhanced given that the allocation of the rate, power and decoding order over the users is optimized. The optimal rate and power allocation for SC with SIC at the receivers was studied in the literature in [5] and [6]. It is not that all users get scheduled over all channel blocks, but rather the number of scheduled users varies dynamically based on the channel conditions of all users such that only the users with good channel conditions get selected. The statistics of the optimal number of scheduled users using SC depend on the users' channels statistics. In this letter, Rayleigh fading channels are considered.

Due to the prospected gains of multiple users scheduling over SU scheduling, there has been some recent work on the performance of multiple users scheduling via SC with SIC at the receivers and its application in the next generation communication standards such as Long Term Evolution (LTE) systems. Two examples of such works are [7] and [8]. However, in these works, the optimal power and rate allocation for SC as studied in [5], [6] was not taken into consideration. Moreover, these works considered the uplink or the multiple access channel (MAC). The optimized SC scheme was considered in [9]. However, the comparison between SU scheduling and SC was done based on the achievable rate region of a two-user scenario. This was insightful, but it cannot be extended into higher number of users exceeding three. Therefore, to compare the two scheduling schemes for higher number of users, other suitable comparison criteria need to be found, which is done in this letter.

The contribution of this letter is in providing new insightful numerical simulations of the optimal SC scheme. In particular, two aspects of the system are considered. The first one is the statistics of the optimal number of scheduled users over Rayleigh fading channels and the statistics of the average channel access ratio of each user. The second one is comparing the long-term average sum rate (i.e. throughput) achieved by the optimal SC scheme and the conventional SU scheduling scheme. The gain of the optimal SC scheme over the SU scheduling scheme is obtained versus the total number of users in the system. To have consistent comparisons, the two scheduling schemes are compared at three different properly defined operating points as explained in more detail in Section III. 


\section{PRELIMinaries}

\section{A. System Model}

Consider a system with one source node $(\mathrm{S})$ and $M$ users or destination nodes (D) yielding a broadcast (i.e. downlink) channel. The information sent for each user is independent of the information sent to other users. It is assumed that all nodes are equipped with a single antenna. The channels between the source and the users are block-fading. Therefore, the time and frequency grid is divided into channel blocks, called resource units (RUs), with a certain time duration and bandwidth. The channel is assumed to be constant over one RU and changes randomly and independently from one RU to another. Therefore, within one RU, the channel between the source and any user is modeled as additive white Gaussian noise (AWGN) channel. Furthermore, the channels of the users are assumed to be independent. Without loss of generality, it is assumed that each RU has the same time duration and bandwidth. Constant power, denoted by $\bar{P}$, per RU is also assumed.

For notation, the index $k$ is used for the RUs. $h_{i}[k]$ is the channel response between the source and the $i$ th user over the $k$ th RU. $\gamma_{i}[k]=\frac{\left|h_{i}[k]\right|^{2} \bar{P}}{N_{0}}$ denotes the Signal-to-NoiseRatio (SNR), where $N_{0}$ is the additive noise power spectral density. $R_{i}[k]$ and $\bar{R}_{i}$ denote respectively the achievable rate (in bits/sec/Hz) by the $i$ th user in the $k$ th $\mathrm{RU}$ and the longterm average (over all RUs) achievable rate (throughput) of that user. Furthermore, the channels of all users are assumed to be Rayleigh block-faded.

It is assumed that the source has full channel state information (CSI) of all channels through feedback. The source incorporates the CSI in the scheduling task in order to obtain MUD gains. The scheduling schemes are optimized to be the solution of the optimization problem

$$
\max \sum_{i=1}^{M} \mu_{i} \bar{R}_{i}=\frac{1}{K} \sum_{i=1}^{M} \mu_{i} \sum_{k=1}^{K} R_{i}[k]
$$

where the total number of RUs, i.e. $K$, approaches infinity and $\mu_{i}$ is the merit weighting factor assigned to the $i$ th user. For the SU scheduling scheme, there is an additional constraint that only a single user can have a non-zero rate per RU. This added constraint makes SU scheduling suboptimal.

\section{B. Single User Selection}

With the SU selection constraint, the solution of (1) yields selecting the scheduled user according to

$$
\zeta[k]=\arg \max _{i} \mu_{i} R_{i}[k] \quad i=1, \ldots, M,
$$

where $\zeta[k]$ is the index of the selected user in the $k$ th RU. Therefore, the achievable rate by SU scheduling is given as

$$
R_{i}[k]= \begin{cases}\log _{2}\left(1+\frac{\bar{P}\left|h_{i}[k]\right|^{2}}{N_{0}}\right) & : i=\zeta[k] \\ 0 & \text { otherwise. }\end{cases}
$$

The average throughput of a user is given by (e.g. [2])

$$
\bar{R}_{i}=\int_{0}^{\infty} r f_{R_{i}}(r) \prod_{l \neq i} F_{R_{l}}\left(\frac{\mu_{i} r}{\mu_{l}}\right) d r .
$$

where $f_{R}(r)$ and $F_{R}(r)$ are respectively the probability density function (PDF) and cumulative distribution function (CDF) of the achievable rate.

The ratio of the number RUs in which a user is scheduled to the total number of RUs, denoted by $\tau_{i}$, is given by

$$
\tau_{i}=\int_{0}^{\infty} f_{R_{i}}(r) \prod_{l \neq i} F_{R_{l}}\left(\frac{\mu_{i} r}{\mu_{l}}\right) d r
$$

\section{Superposition Coding with Successive Interference Cancel- lation For Decoding}

A procedure to optimize the power and rate allocation for the SC based on a greedy algorithm that involves marginal utility functions for every user was suggested in [6] for the case of variable power allocation over the channel blocks (i.e. RUs). The straightforward extension to the constant power per RU case was outlined in [9]. The latter case is summarized as follows; The marginal utility functions in the $k$ th RU are defined for every user as

$$
u_{i}(k, z) \equiv \frac{\mu_{i}}{\frac{1}{\left|h_{i}[k]\right|^{2}}+z}, \quad 0 \leq z \leq \frac{\bar{P}}{N_{0}},
$$

where $z$ refers to the interference levels. The rate (in bits/sec/Hz) and power (in Joul/sec/Hz) allocated to each user depends on the marginal utility functions according to

$$
R_{i}[k]=\frac{1}{\ln 2} \int_{\mathbb{A}_{i}[k]} \frac{1}{\frac{1}{\left|h_{i}[k]\right|^{2}}+z} d z, \quad P_{i}[k]=N_{0} \int_{\mathbb{A}_{i}[k]} d z,
$$

where the period $\mathbb{A}_{i}[k]$ is defined as

$$
\mathbb{A}_{i}[k] \equiv\left\{z \in\left[0, \frac{\bar{P}}{N_{0}}\right]: u_{i}(k, z)>u_{j}(k, z) \forall j \neq i\right\} .
$$

Notice that if $\mathbb{A}_{i}[k]$ is an empty set, then the $i$ th user is not scheduled in the corresponding RU. The users' order in the SIC process is in the order of decreasing $\mu$, meaning that each user decodes the codewords of users who have higher $\mu$ 's before decoding its own codeword.

The long term throughput of the $i$ th user is given by ${ }^{1}$ [9]

$$
\begin{aligned}
\bar{R}_{i}= & \int_{0}^{1} \int_{0}^{\infty} \frac{1}{\frac{1}{\gamma}+z} f_{\gamma_{i}}(\gamma) . \\
& \prod_{j \neq i} F_{\gamma_{j}}\left(\left[\frac{\mu_{i} \gamma}{\mu_{j}+\mu_{j} \gamma z-\mu_{i} \gamma z}\right]^{*}\right) d \gamma d z,
\end{aligned}
$$

where the notation $[x]^{*}$ is defined as [10]

$$
[x]^{*}= \begin{cases}\infty & : x<0 \\ x & \text { otherwise. }\end{cases}
$$

The ratio of the number of RUs in which the $i$ th user is scheduled (channel access ratio) can be found by

$$
\tau_{i}=\int_{0}^{1} \int_{0}^{\infty} f_{\gamma_{i}}(\gamma) \prod_{j \neq i} F_{\gamma_{j}}\left(\left[\frac{\mu_{i} \gamma}{\mu_{j}+\mu_{j} \gamma z-\mu_{i} \gamma z}\right]^{*}\right) d \gamma d z
$$

\footnotetext{
${ }^{1}$ An equivalent expression to the one in [9] is used since here the PDF and CDF of SNR $\left(\gamma=|h|^{2} \bar{P} / N_{0}\right)$ are required, while in [9] the PDF of $|h|^{2}$ is used.
} 


\section{CRiteria For The COMPARISONS BETWEen SCHEDULING SCHEMES}

For the simulation framework, the following model for the system is used. It is assumed that the users are uniformly distributed in a circular region around the base station between two bounds $d_{\min }$ and $d_{\max }$ for the minimum and maximum distance from the base station. Therefore, the PDF and CDF for the distance of a user from the base station is given by

$$
f_{D}(d)=\frac{2 d}{d_{\max }^{2}-d_{\min }^{2}}, \quad F_{D}(d)=\frac{d^{2}-d_{\min }^{2}}{d_{\max }^{2}-d_{\min }^{2}}
$$

Let's assume that there are $M$ users in the cell. The distances of the users are obtained randomly using (12) and then they are arranged in descending order, denoted by $d_{1}, d_{2}, \cdots, d_{M}$ from the maximum to the minimum distance. The expected values of the ordered distances are obtained by

$$
\bar{d}_{i}=i\left(\begin{array}{c}
M \\
i
\end{array}\right) \int_{d_{\min }}^{d_{\max }} x f_{D}(x) F_{D}(x)^{M-i}\left(1-F_{D}(x)\right)^{i-1} d x
$$

From the expected values for the distances, we obtain the expected values for SNR, arranged in ascending order, according to the model $\left(\bar{\gamma}_{i}=\beta\left(\bar{d}_{i}\right)^{-\alpha}\right)$, where $\alpha$ is the path loss exponent and $\beta$ is a constant related to the transmission power and antenna gains. In our simulations, we use $\alpha=3$, $\beta=10^{3}, d_{\min }=1$ and $d_{\max }=10$. Therefore, the average SNR ranges between $0 \mathrm{~dB}$ at $d_{\max }$ and $30 \mathrm{~dB}$ at $d_{\min }$.

We compare between SC and SU selection for variable number of users. For a given number of users, we generate the expected SNR values of the users using the described model for uniform distribution in a circular cell. Furthermore, we compare the scheduling schemes for three different and well-defined optimization functions in order to have a broader picture of the gains of SC at different operating points of the system. The challenging task is to obtain the users' weighting factors (i.e. $\mu$ ) for every case.

\section{A. Hard Fairness - Equal Rates}

In this case, the optimization objective function is

$$
\max \min \left(\bar{R}_{i}, i \in\{1,2, \cdots, M\}\right)
$$

The optimal solution is achieved when all users have equal throughput on the long-term $\left(\bar{R}_{i}=\bar{R}_{j} \forall i, j\right)$. The problem formulation in (14) is equivalent to (1) with the users' weights properly selected to make the average rates of the users equal. A numerical method is applied to obtain the users' weights within a small tolerance value from the equal rates point, as described in Algorithm 1. $\delta$ controls the speed of convergence. Notice that the weights to achieve hard fairness in $\mathrm{SU}$ selection is different from the SC case.

\section{B. Proportional Fairness}

In this case, the optimization objective function is

$$
\max \left(\prod_{i=1}^{M} \bar{R}_{i}\right)^{1 / M}
$$

It can be shown that (15) is equivalent to (1) with the weight of each user selected to be the reciprocal of its average

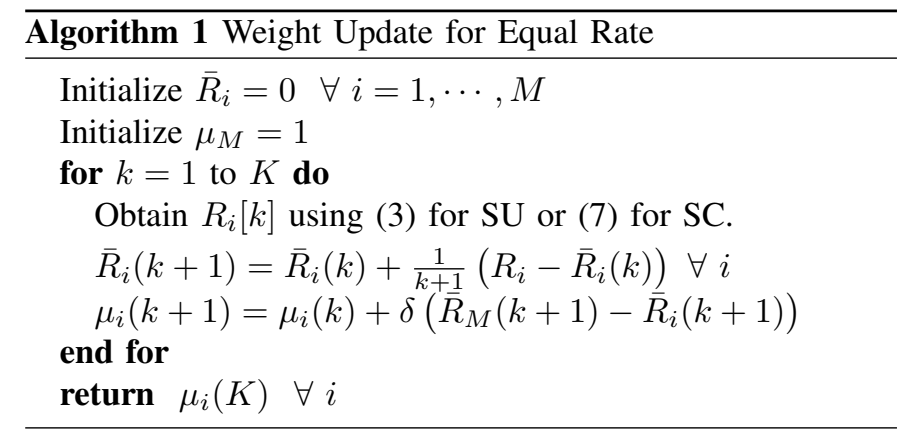

throughput $\left(\mu_{i}=1 / \bar{R}_{i}\right)$ [11]. Since the average throughputs cannot be known in advance, they are obtained using a recursive algorithm such that the weights and throughputs are continuously updated until they converge into their steady state values. Such an algorithm was applied in [12].

\section{Fixed Pre-determined Users' Weighting Factors}

In this case, we apply (1) directly and use the same users' weights for both SU selection and SC. We selected the weights arbitrary to be the reciprocal of the achievable rate at the average SNR for each user $\left(\mu_{i}=1 / \log _{2}\left(1+\bar{\gamma}_{i}\right)\right)$.

\section{Numerical Results And Conclusions}

Fig. 1 shows the comparison between SU selection and SC in terms of the long term sum throughput plotted versus the total number of users in the system for the system model described in Section III. As expected, for both SC and SU selection, the sum throughput increases as the number of users increases due to the MUD gains, and SC achieves higher sum throughput than SU selection. To quantify the gain of SC, we show in Fig. 2 the ratio between SC and SU selection in terms of the maximum achievable values for the objective functions (14), (15) and (1), for hard fairness, proportional fairness and equal weights, respectively using three different network models ${ }^{2}$. A new learned conclusion is that the gain of SC over SU selection increases as the number of users increases. This is true for the three different operating points which are investigated in this work. Another important conclusion is that the gain of SC can exceed $10 \%$ for high number of users. Furthermore, the gain of $\mathrm{SC}$, as a function of the number of users, is to a big extent similar for the three optimization functions although they reflect three different operating points in terms of the trade-off between total throughput and fairness.

Fig. 3 shows the channel access ratio for the individual users in a ten-user scenario. As expected, the channel access ratio with SC is greater for each user than SU selection. This is due to the fact that multiple users can be scheduled in the same RU. Another important observation is that with SC, the users with worst and best channel conditions are scheduled more frequently than the remaining users. Notice that this is not the case with SU selection.

\footnotetext{
${ }^{2}$ To give a more comprehensive analysis, two additional models to obtain the average SNR of the users are used for Fig. 2. In the first model, the SNRs of the best and worst channels are similar to the original model while the SNRs in between are distributed uniformly between these two limits. In the second model, we use our original model with a smaller range between minimum and maximum SNR values, which are changed to $5 \mathrm{~dB}$ and $25 \mathrm{~dB}$, respectively.
} 


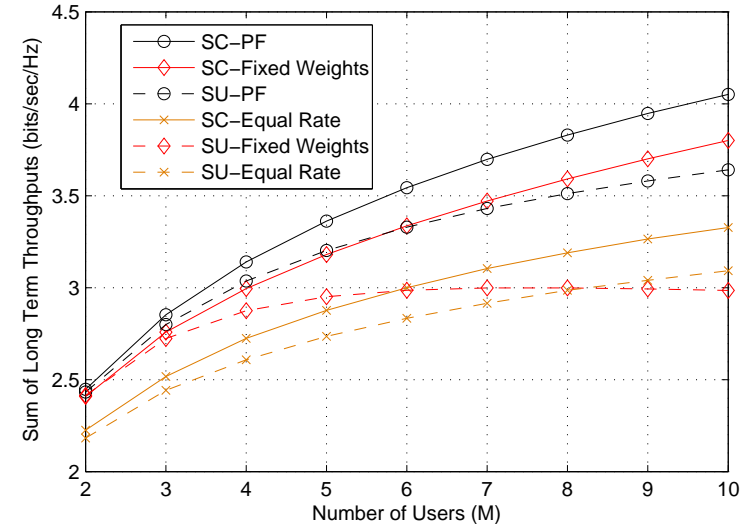

Fig. 1: Comparison of SC and SU in term of sum of long term throughputs of all the users.

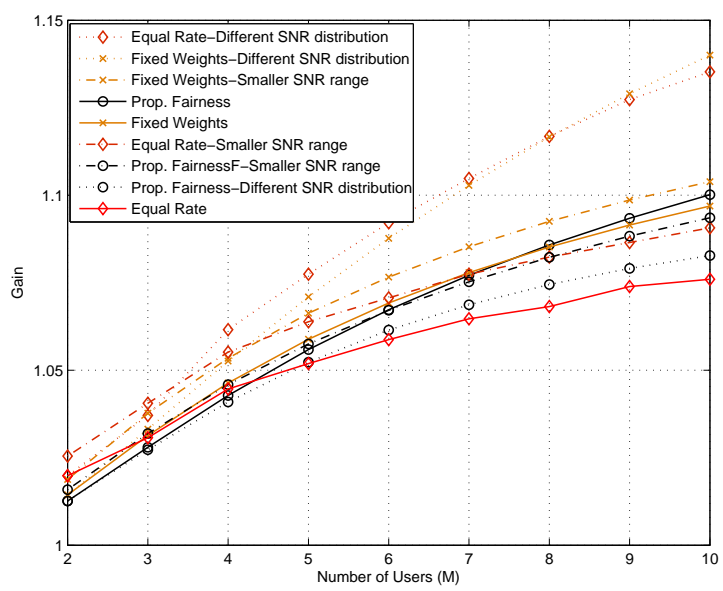

Fig. 2: Gain in performance of SC over SU as a function of number of users, $M$.

The statistics of the number of scheduled users per RU for $\mathrm{SC}$ at proportional fairness are shown in Fig. 4 for number of users $M=10, M=6$ and $M=3$. We found that the statistics have a very similar pattern for hard fairness as well as fixed weights case. Therefore, their results are not shown. An important learned conclusion from Fig. 4 is that even if the number of users is high, e.g. 10, we have high probability that a limited number of users are scheduled in each $R U$. We could even have a single user scheduling, but it has low probability of occurring. The expected number of scheduled users using SC is important when we consider the practical implementation of the scheme due to the complexity constraints. Therefore, in our opinion, $10 \%$ increase in throughput for two or three layers of superposition coding is a good compromise between prospected gains and added complexity. Therefore, we believe that superposition coding has the potential to be a good option for practical implementation in next generation systems.

\section{REFERENCES}

[1] D. Tse and P. Viswanath, Fundamentals of Wireless Communication. Cambridge UniversityPress, 2005.

[2] M. Shaqfeh and N. Goertz, "Performance analysis of scheduling policies for delay-tolerant applications in centralized wireless networks," in Proceedings SPECTS'08, Edinburgh, UK, Jun. 2008, pp. 309-316.

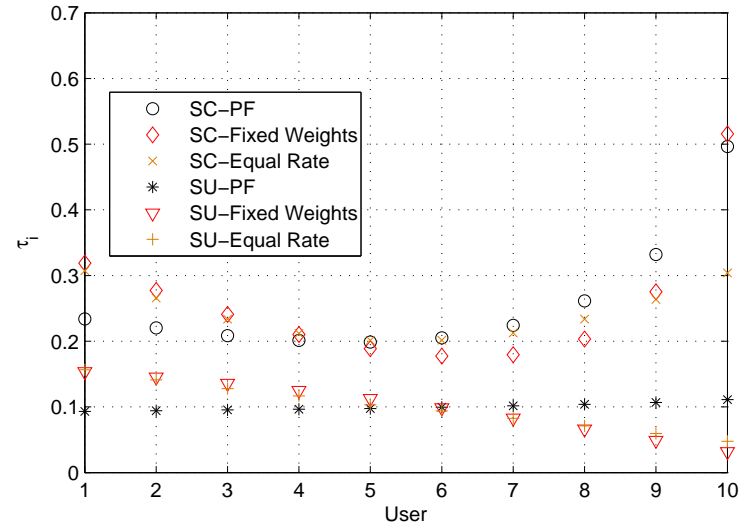

Fig. 3: Channel access ratio of each user for a system with 10 users. The average SNR, $\bar{\gamma}$, increases with the increase in user index. Therefore, user 10 has the highest SNR.
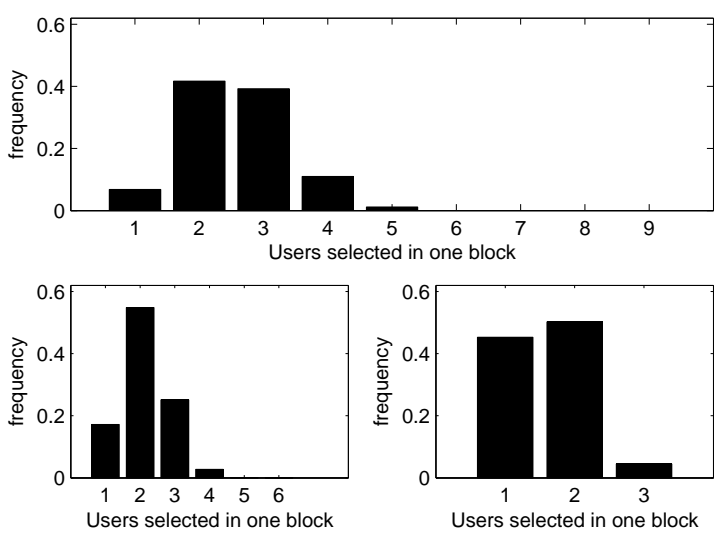

Fig. 4: Bar plot of number of users selected in one RU for a system with 10,6 and 3 users respectively.

[3] R. Knopp and P. A. Humblet, "Information capacity and power control in single-cell multiuser communications," in Proceedings ICC'95, Seattle, WA, USA, Jun. 1995, pp. 331-335.

[4] T. Cover and J. Thomas, Elements of Information Theory, 2nd ed. Wiley-Interscience, July 2006.

[5] L. Li and A. Goldsmith, "Capacity and optimal resource allocation for fading broadcast channels. i. ergodic capacity," IEEE Transactions on Information Theory, vol. 47, no. 3, pp. 1083-1102, Mar. 2001.

[6] D. N. C. Tse, "Optimal power allocation over parallel Gaussian broadcast channels," available: www.eecs.berkeley.edu/ dtse/broadcast2.pdf.

[7] J. Schaepperle, "Throughput of a wireless cell using superposition based multiple-access with optimized scheduling," in Proceedings PIMRC'2010, Istanbul, Turkey, Sep. 2010, pp. 212-217.

[8] N. Prasad, H. Zhang, H. Zhu, and S. Rangarajan, "Multi-user scheduling in the 3GPP LTE cellular uplink," in Proceedings WiOpt'2012, Paderborn, Germany, May 2012, pp. 262-269.

[9] M. Shaqfeh, N. Goertz, and J. Thompson, "Ergodic capacity of blockfading Gaussian broadcast and multi-access channels for single-userselection and constant-power," in Proceedings EUSIPCO'09, Glasgow, Scotland, UK, Aug. 2009, pp. 784-788.

[10] M. Shaqfeh and N. Goertz, "Comments on the boundary of the capacity region of multiaccess fading channels," IEEE Transactions on Information Theory, vol. 55, no. 7, pp. 3407-3408, Jul. 2009.

[11] P. Viswanath, D. Tse, and R. Laroia, "Opportunistic beamforming using dumb antennas," IEEE Transactions on Information Theory, vol. 48, no. 6, pp. 1277-1294, Jun. 2002.

[12] A. Zafar, M. Shaqfeh, M.-S. Alouini, and H. Alnuweiri, "Joint scheduling for dual-hop block-fading broadcast channels," in Proceedings 8th IEEE BWA Workshop, co-located with IEEE GLOBECOM 2012, Anaheim, CA, Dec. 2012, available at: http://hdl.handle.net/10754/244151. 